\title{
MODELLING OF NONLINEAR CROSSTALK IN OPTICAL FIBRE WAVELENGTH-DIVISION -MULTIPLEXED TELECOMMUNICATION SYSTEMS
}

\author{
W. SZCZg̨SNy AND M. MarCiniak \\ Institute of Telecommunications, Department of Transmission and Fibre Technology \\ Szachowa 1, 04-894 Warsaw, Poland
}

\begin{abstract}
Nonlinear effects in optical fibres play an important role for transmission parameters in wavelength-division multiplexed telecommunication systems. Those effects cause an additional attenuation, distortion and inter-channel interference of the multi-wavelength optical signal. Therefore, it is important to optimise inter-channel wavelength span in a wavelength-division multiplexed system, and also the optical power and transmission bit-rate. Another drawback is a limitation of the number of the transmitted optical channels and network nodes. The paper reports the results of the analysis and computer simulations of the wavelength-division multiplexed transmission through fibre link in a presence of nonlinear effects: self-phase modulation, cross-phase modulation, Raman scattering, four-wave mixing. The analysis enables network designers to evaluate the system performance and to optimise transmission parameters.
\end{abstract}

PACS numbers: 42.81.-i, 42.79.Sz

\section{Introduction to WDM systems}

Recent years have shown a rapid growth of demand for capacity of telecommunication networks. It has inspired many laboratories to explore new techniques of more efficient utilisation of the huge bandwidth offered by optical fibre links. One of the most promising and cost effective ways to increase optical link throughput is a technique known as wavelength-division multiplexing (WDM).

In a WDM system many information channels are transmitted through one fibre using different optical wavelengths modulated by independent data streams. This method is analogous to frequency division multiplexing (FDM) which is widely exploited in other communication systems, especially in radio broadcasting. Using WDM we can easily increase the capacity of already existing fibre links that is particularly significant in the areas where placing of new cables is impossible or too expensive. WDM is a technique compatible with the idea of all-optical networks, where one can create transparent optical paths connecting successive 
network nodes by switching optical channels organised at the different light wavelengths. One can also envision the application of WDM in broadcast networks and/or in subscriber loop [1].

These and other advantages of WDM have prompted the beginning of standardisation work [2]. Nevertheless, the job is not yet completed and further research and estimations are required [3].

\section{Nonlinear limitations}

In spite of its merits the WDM technique is not free from limitations. The most characteristic and essential problem for multichannel optical systems, beside attenuation and dispersion, is interchannel crosstalk [4]. One can distinguish crosstalk caused by nonlinear interactions between the light in different channels or between the light and the fibre material. In spite of the intrinsically small values of the nonlinearity coefficients in fused silica, the nonlinear effects in optical fibres can be observed at low power levels. This is possible because of important characteristics of single-mode fibres, a very small optical beam spot size, and extremely low attenuation $[5,6]$.

In WDM systems a nonlinear interplay between many different spectral components of the aggregate signal causes interchannel crosstalk. The nonlinear phenomena involved are: self-phase modulation (SPM), cross-phase modulation (XPM), four-wave mixing (FWM), stimulated Raman scattering (SRS), and stimulated Brillouin scattering (SBS).

\subsection{Nonlinear refraction}

Most of the nonlinear effects in optical fibres originate from nonlinear refraction, a phenomenon that refers to the intensity dependence of the refractive index resulting from the contribution of $\chi^{(3)}$, i.e., the refractive index of the fibre becomes

$$
\tilde{n}\left(\omega,|\boldsymbol{E}|^{2}\right)=n(\omega)+n_{2}\left(|\boldsymbol{E}|^{2}\right)
$$

where $n(\omega)$ is the linear part, $|\boldsymbol{E}|^{2}$ is the optical intensity inside the fibre, and $n_{2}$ is the nonlinear-index coefficient related to $\chi^{(3)}$ by the relation [2]

$$
n_{2}=\frac{3}{8 n(\omega)} \operatorname{Re}\left(\chi_{x x x}^{(3)}\right)
$$

where Re stands for the real part and the optical field is assumed to be linearly polarised so that only one component of the $\chi_{x x x}^{(3)}$ fourth-rank tensor contributes to the refractive index. The intensity dependence of the refractive index leads to a large number of interesting nonlinear effects; the two most widely studied are: self-phase modulation and cross-phase modulation.

\subsection{Self-phase modulation}

SPM refers to the self-induced phase shift experienced by an optical field during its propagation in optical fibres. Its magnitude can be obtained by noting that the phase of an optical field changes by

$$
\phi=\tilde{n} k_{0} L=\left[n(\omega)+n_{2}|\boldsymbol{E}|^{2}\right] k_{0} L,
$$

where $k_{0}=2 \pi / \lambda$ and $L$ is the fibre length. The intensity-dependent nonlinear phase shift $\phi_{\mathrm{NL}}=n_{2} k_{0} L|\boldsymbol{E}|^{2}$ is due to SPM. The phase shift (3) is frequency dependent due to the chromatic dispersion of the fiber. 


\subsection{Cross-phase modulation}

XPM refers to the nonlinear phase shift of an optical field induced by a co-propagating field at different wavelengths. The total electric field $\boldsymbol{E}$ is given by

$$
\boldsymbol{E}=\frac{1}{2} \widehat{x}\left[E_{1} \exp \left(-\mathrm{i} \omega_{1} t\right)+E_{21} \exp \left(-\mathrm{i} \omega_{2} t\right)+\text { c.c. }\right] \text {. }
$$

When two optical fields at frequencies $\omega_{1}$ and $\omega_{2}$, polarised along the $x$-axis, co-propagate simultaneously inside the fibre, the nonlinear phase shift for the field at $\omega_{1}$ is given by

$$
\phi_{\mathrm{NL}}=n_{2} k_{0} L\left(\left|\boldsymbol{E}_{1}\right|^{2}+2\left|\boldsymbol{E}_{2}\right|^{2}\right) \text {, }
$$

where we have neglected all terms that generate polarisation at frequencies other than $\omega_{1}$ and $\omega_{2}$ because of their non-phase-matched character. The two terms on the right hand side of Eq. (5) are due to SPM and XPM, respectively. An important characteristic of XPM is that, for equally intense optical fields, the contribution of XPM to the nonlinear phase shift is twice compared with that of SPM. Among other things, XPM is responsible for asymmetric spectral broadening of co-propagating optical pulses.

In a WDM system the total phase shift depends on the power in all channels and varies from bit to bit depending on the bit pattern of the neighbouring channels.

The phase modulation may cause an increase in crosstalk in WDM systems. This is especially expected in systems with planar arrayed-waveguide grating (AWG) demultiplexers, which exhibit low selectivity. For synchronous transmission systems SDH, SPM, and XPM can lead to de-synchronisation of the aggregate optical signal due to temporal changes in phase relations for optical pulses in different channels. Also XPM and SPM enhance the efficacy of FWM, which is another cause of degradation of WDM system transmission performance.

\subsection{Stimulated scattering - Raman and Brillouin}

The nonlinear effects governed by the third-order susceptibility $\chi^{(3)}$ are elastic in the sense that no energy is exchanged between the electromagnetic field and the dielectric medium. A second class of nonlinear effects results from stimulated inelastic scattering in which the optical field transfers a part of its energy to the nonlinear medium. Two important nonlinear effects in optical fibres fall in this category; both of them are related to vibrational excitation modes of silica. These phenomena are known as stimulated Raman scattering (SRS) and stimulated Brillouin scattering (SBS) and were among the first nonlinear effects studied in optical fibres. The main difference between them is that optical phonons participate in SRS while acoustic phonons participate in SBS. In a simple quantum-mechanical picture applicable to both SRS and SBS, a photon of the incident field (often called the pump) is annihilated to create a photon at the downshifted Stokes frequency and a phonon with the right energy and momentum to conserve the energy and the momentum. Of course, a higher-energy photon at the so-called anti-Stokes frequency can also be created if a phonon of right energy and momentum is available. Even though SRS and SBS are very similar in their origin, different dispersion relations for acoustic and optical phonons lead to some basic differences between 
the two. A fundamental difference is that SBS in optical fibres occurs only in the backward direction whereas SRS dominates in the forward direction.

The initial growth of the Stokes wave can be described by a simple relation. For SRS, this relation is given by [2]

$$
\frac{\mathrm{d} I_{\mathrm{S}}}{\mathrm{d} z}=g_{\mathrm{R}} I_{\mathrm{P}} I_{\mathrm{S}},
$$

where $I_{\mathrm{S}}$ is the Stokes intensity, $I_{\mathrm{P}}$ is the pump intensity, and $g_{\mathrm{R}}$ is the Raman-gain coefficient. A similar relation holds for SBS with $g_{\mathrm{R}}$ replaced by the Brillouin-gain coefficient $g_{\mathrm{B}}$. Both $g_{\mathrm{R}}$ and $g_{\mathrm{B}}$ have been measured experimentally for silica fibres. The Raman-gain spectrum is measured to be very broad extending up to $\sim 30 \mathrm{THz}$. The peak gain $g_{\mathrm{R}}=10^{-13} \mathrm{~m} / \mathrm{W}$ at a pump wavelength of $1 \mu \mathrm{m}$ and occurs at the Stokes shift of about $13 \mathrm{THz}$.

By contrast, the Brillouin-gain spectrum is extremely narrow with a bandwidth $\sim 10 \mathrm{MHz}$. The peak value of Brillouin gain occurs at the Stokes shift $\sim 10 \mathrm{GHz}$. The peak gain is about $6 \times 10^{-11} \mathrm{~m} / \mathrm{W}$ for a narrow-bandwidth pump and decreases by a factor of $\Delta \nu_{\mathrm{P}} / \Delta \nu_{\mathrm{B}}$ for a broad-bandwidth pump, where $\Delta \nu_{\mathrm{P}}$ is the pump bandwidth and $\Delta \nu_{\mathrm{B}}$ is the Brillouin-gain bandwidth.

The influence of SBS can be neglected in real WDM systems with inter-channel spacing $\geq 100 \mathrm{GHz}$ and transmission speed $\geq 1 \mathrm{GHz}$, because $\Delta \nu_{\mathrm{P}} \gg \Delta \nu_{\mathrm{B}}$ in this case. However, one should take SBS into account for inter-channel crosstalk and loss estimation. In a two-channel system, if we inject two optical waves separated by the Stokes frequency into the fibre where Raman interactions take place, the power of the lower frequency wave (called the probe) will increase in the expense of the higher frequency wave (called the pump). Such an energy transfer from one channel to another is called inter-channel crosstalk. It is important to underline that SRS appears when the light is present in both channels, i.e. "1" bits are transmitted simultaneously.

The situation is more complicated for multi-channel systems. The intermediate channels not only transfer energy to the longer-wavelength channels but, at the same time, also receive energy from the shorter-wavelength channels.

A simplified model of optical power losses in a multi-channel WDM system has been given in Ref. [7]:

$$
P_{\text {-rem }}=-10 \log (1-D) \text {, }
$$

where

$$
D=\sum_{i=1}^{N-1} \frac{f_{0} P_{i} g_{i} L_{\mathrm{eff}}}{f_{i} A_{\mathrm{eff}}}
$$

$P_{-}$rem - the fraction of the power that remains at the shortest-wavelength channel, $i$ - channel index, $f_{0}$ - frequency of the 0-th channel, $f_{i}$ - frequency of the $i$-th channel, $N$ - number of channels, $P_{i}$ - power injected in the $i$-th channel, $L_{\text {eff }}-$ effective fibre length, $A_{\text {eff }}-$ effective core area, $g_{i}$ - Raman gain coefficient coupling the $i$-th channel with the 0 -th channel.

The model assumes that walk-off length $L_{\mathrm{W}}$ is much longer than the actual fibre length $L$. However, for digital systems with a high transmission rate $(<1 \mathrm{~Gb} / \mathrm{s})$ and short pulse duration $(\sim 100 \mathrm{ps})$ usually $L_{\mathrm{W}}<L$. In this case 
SRS is limited by the group-velocity mismatch and occurs only over distances $z \sim L_{\mathrm{W}}$ even if the actual fibre length $L$ is considerably larger than $L_{\mathrm{W}}$. At the same time, nonlinear effects such as SPM and XPM become important because of relatively large peak powers and considerably affect evolution of the pump and Raman pulses.

In the WDM the depletion introduced by SRS has an unfortunate characteristic: The depletion of a given " 1 " is dependent on the signals that are other channels. This means that the "drops" caused by SRS can occur on the timescale of an individual bit, which is much faster than the response time of the threshold setting in the receiver. The depth and the frequency of these drops will dictate the bit-error ratio (BER) for the WDM system that is limited by SRS [2].

\subsection{Four-wave mixing}

Four-wave mixing, a nonlinear phenomenon, is known to affect the SRS in any medium, also in optical fibres. Raman scattering can be thought of as a down-conversion of a pump photon into a lower-frequency photon and a phonon associated with a vibrational mode of molecules. An up-conversion process in which a phonon combines with the pump photon to generate a higher-frequency photon is also possible, but occurs rarely because it requires the presence of a phonon of right energy and momentum. The optical wave associated with higher-frequency photons is called anti-Stokes and is generated at a frequency $\omega_{\mathrm{a}}=\omega_{\mathrm{p}}+\Omega$ for a Stokes wave of frequency $\omega_{\mathrm{s}}=\omega_{\mathrm{p}}+\Omega$, where $\omega_{\mathrm{p}}$ is the pump frequency. Since

$$
2 \omega_{\mathrm{p}}=\omega_{\mathrm{a}}+\omega_{\mathrm{s}}
$$

four-wave mixing, where two pump photons annihilate themselves to produce. Stokes and anti-Stokes photons, can occur provided the total momentum is conserved. The momentum-conservation requirement leads to the phase-matching condition,

$$
\Delta k=2 k_{\mathrm{p}}-k_{\mathrm{a}}-k_{\mathrm{s}}=0,
$$

that must be satisfied for four-wave mixing to take place. The phase-matching condition is not easily satisfied in single-mode fibres for $\Omega \sim 10 \mathrm{THz}$. For this reason, the anti-Stokes wave is rarely observed during SRS.

The efficiency of FWM can be large when two or more different wavelengths propagate simultaneously in the fibre. When optical pulsations involved are $\omega_{1}$ and $\omega_{2}, \omega_{2}>\omega_{1}$, then, according to (9), new pulsations $\omega_{4}=2 \omega_{1}+\omega_{2}$ and $\omega_{3}=2 \omega_{2}+\omega_{1}$ (for $\omega_{4}<\omega_{3}$ ) are generated. Efficiency of this phenomenon is characterised by parametric gain coefficient $g$ [2]

$$
g=\left[\left(\gamma P_{0} r\right)^{2}-\left(\frac{\kappa}{2}\right)^{2}\right]^{1 / 2}
$$

where

$$
\begin{aligned}
& \kappa=\Delta k+\gamma\left(P_{1}+P_{2}\right), \\
& \Delta k=k_{3}+k_{4}-k_{1}-k_{2}=\frac{\tilde{n}_{3} \omega_{3}+\tilde{n}_{4} \omega_{4}-\tilde{n}_{1} \omega_{1}-\tilde{n}_{2} \omega_{2}}{c},
\end{aligned}
$$




$$
\begin{aligned}
& r=2 \frac{\left(P_{1} P_{2}\right)^{1 / 2}}{P_{0}} \\
& P_{0}=P_{1}+P_{2}
\end{aligned}
$$

and nonlinearity coefficient

$$
\gamma \approx \frac{n_{2} \omega}{c A_{\text {eff }}}
$$

It follows from (11) that FWM can occur with no phase matching in the case of two or more co-propagating wavelengths, it is maximum when the so-called mis-matching coefficient $\kappa=0$. This coefficient in a single-mode fibre is given approximately by $\kappa \approx \beta_{2} \Omega_{\mathrm{S}}^{2}$, where $\beta_{2}$ is the pump wave $\left(\omega_{1}\right)$ dispersion coefficient, and $\Omega_{\mathrm{S}}$ is the frequency shift. Consequently, FWM efficiency is large for standard fibres at $1.3 \mu \mathrm{m}$ and for dispersion-shifted fibres at $1.55 \mu \mathrm{m}$, even for a small pump power. That means also generation of inter-channel crosstalk in equidistantly spaced WDM systems.

A simple analysis based on continuous-wave (cw) assumption reported above allows only for a qualitative description of the impact of nonlinear phenomena on WDM transmission. Actually, all nonlinear effects influence themselves in the fibre. Also, the cw model is not very suitable for high bit-rate transmission systems. On the other hand, the knowledge of crosstalk is very important in practical systems. E.g. in SDH systems even low-level crostalk of $-25 \mathrm{~dB}$ causes a considerable degradation of signal-to-noise $(\mathrm{S} / \mathrm{N})$ ratio and bit-error rate. Therefore, a more exact analysis is needed. It is based on numerical solutions of wave equation in a nonlinear fibre.

Propagation of the field in the $X$-polarisation state in a nonlinear fibre is modelled using the following nonlinear partial differential equation [4]:

$$
\frac{\partial A_{x}}{\partial z}-\frac{\mathrm{i}}{2} \beta_{2} \frac{\partial^{2} A_{x}}{\partial T^{2}}-\frac{1}{6} \beta_{3} \frac{\partial^{2} A_{x}}{\partial T^{2}}+\frac{\alpha}{2} A_{x}=-\mathrm{i} \gamma\left[\left|A_{x}\right|^{2} A_{x}-T_{\mathrm{R}} A_{x} \frac{\partial^{2}\left|A_{x}\right|^{2}}{\partial T^{2}}\right]
$$

where $A_{x}(z, T)$ is the slowly varying field envelope and $\beta_{2}, \beta_{3}, \alpha$, and $\gamma$ are related to the dispersion, dispersion slope, loss, and nonlinearity of the fibre, respectively. $T_{R}$ is related to the slope of the Raman gain and is assumed to vary linearly with frequency in the vicinity of the carrier frequency [8]. The parameter $T_{R}$ is estimated to be $\sim 5 \mathrm{fs}[8]$. The Raman gain is polarisation dependent, and consequently, the value of $T_{R}$ has to be halved from its value for identical states of polarisation if one wants to account for the effective SRS effect between WDM channels that will have their states of polarisation randomly scrambled at long distances. Equation (17) is solved using the split step Fourier method [4]. The algorithm uses an adaptive step size [8]. The coefficient $\gamma$ accounts for the effects of SPM [9], XPM [3], and FWM [8].

\section{Description of the system analysed}

The simulations have been carried out for an 8-channel STM-64 transmission system, with $10 \mathrm{Gbit} / \mathrm{s}$ single channel data flow, which means an $80 \mathrm{Gbit} / \mathrm{s}$ aggregate transmission speed. The system is shown in Fig. 1 and corresponds to synchronous digital hierarchy (SDH) standards [10]. 


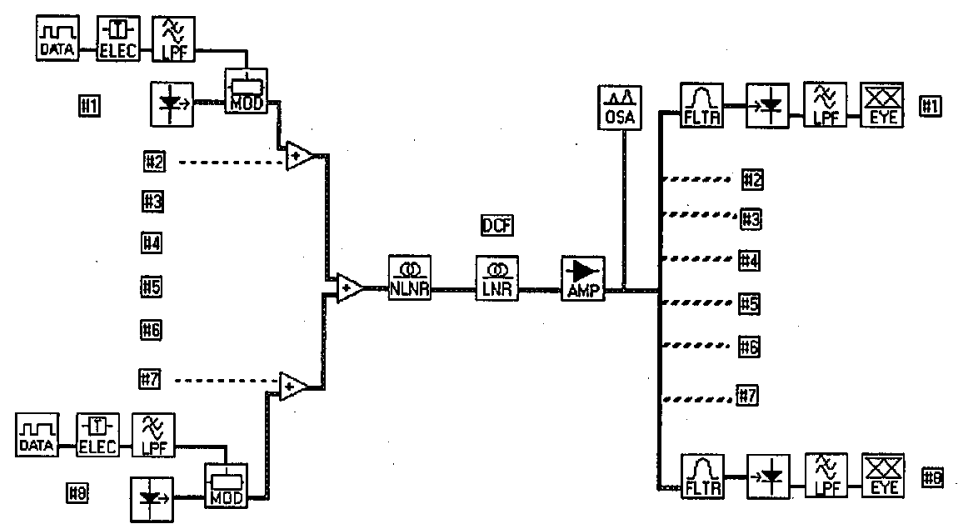

Fig. 1. Architecture of the 8-channel WDM system analysed.

The inter-channel data synchronisation has been obtained as an identical 32-bit pseudo-random sequence in each of WDM channels. De-correlation of the data has been achieved by imposing time delays for varying from channel to channel. At the detector side of the system optical band-pass filters with FWHM $40 \mathrm{GHz}$ bandwidth and different transmission characteristics have been used in order to receive de-multiplex optical channels. The dispersion-shifted fibre is compatible with ITU-T Recommendation G.653 standard [11]. The transmission span is $120 \mathrm{~km}$. A dispersion-compensating fibre with negative dispersion value is introduced at the end of the transmission span. The optical gain in erbium-doped fibre amplifiers has been chosen in a way to compensate for total attenuation of the link. The optical frequency distribution is based on the $100 \mathrm{GHz}(\sim 0.8 \mathrm{~nm})$ grid of ITU-T Recommendation G.692 [12]. The reference frequency for the first WDM channel is $196.1 \mathrm{THz}(1552.52 \mathrm{~nm})$.

Transmitted channels and corresponding frequencies are reported in Table.

TABLE

Frequencies of transmitted channels.

\begin{tabular}{c|c|c|c|c|c|c|c}
\hline \hline \multicolumn{7}{c}{ Channel index } \\
\hline 0 & 1 & 2 & 3 & 4 & 5 & 6 & 7 \\
\hline 196.1 & 196.0 & 195.9 & 195.8 & 195.7 & 195.6 & 195.5 & 195.4
\end{tabular}

A total 8-channel optical power at the transmitter side is $17 \mathrm{dBm}$, this corresponds to laser peak-power of $12.5 \mathrm{~mW}$ and $6.25 \mathrm{~mW}$ mean-power for a non-return to zero (NRZ) code.

\section{Results}

In Fig. 2 there are shown the results of numerical simulations of FWM crosstalk in an equally-spaced 8 channel WDM system with optical signal in all channels (Fig. 2a) and when there is no signal in channel no. 6 (Fig. 2b). The 


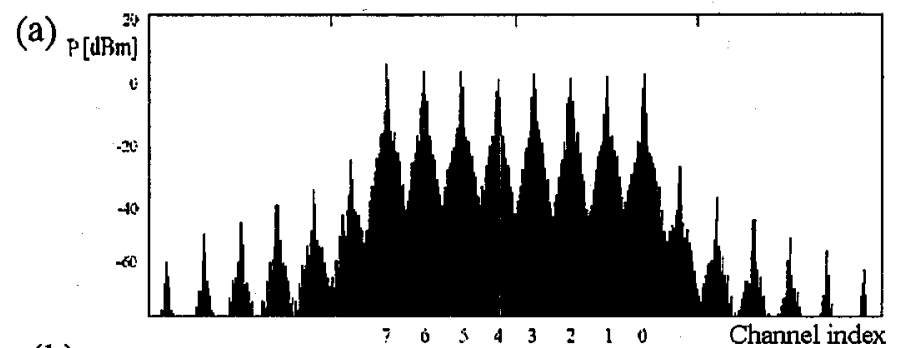

(b)

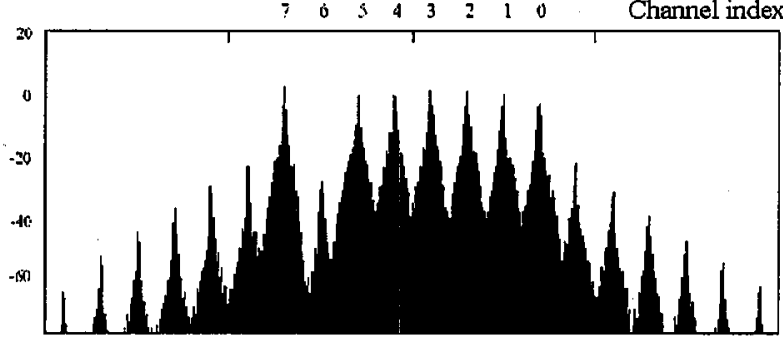

Fig. 2. (a) Spectrum of an 8-channel WDM system when all channels are transmitted and (b) with the absence of channel no. 6, only FWM components are in this channel with a $-24 \mathrm{dBm}$ crosstalk, which is too high for $\mathrm{SDH}$ systems.

(a)
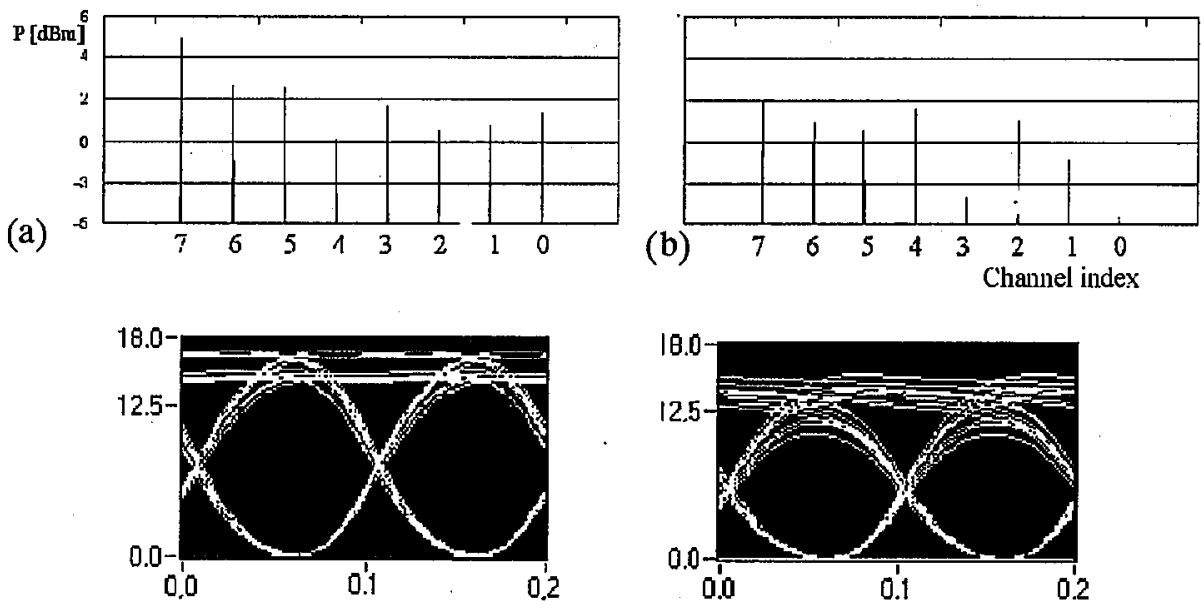

Fig. 3. Influence of Raman scattering on optical power losses in WDM channels. Spectrum of the WDM signal with identical and correlated data stream at the input of the system (a) and the same with identical but no correlated data at the input (b). Maximum power changes of $5 \mathrm{~dB}$ occur in the case (a). Eye diagrams for 0 -th channel show the dependence of temporal pulse shape on data pattern due to SPM and XPM.

presence of FWM products is evident in both figures. Moreover, a large crosstalk in the absent channel no. 6 appears (Fig. 2b).

The results for SRS are reported in Fig. 3, for the WDM signal with identical and correlated data stream at the input of the system (Fig. 3a), and for the signal with identical but no correlated data at the input (Fig. 3b). It can be seen that SRS causes a significant reduction of eye opening (Fig. $3 \mathrm{~b}$, for 0 -th channel). 
The results of XPM crosstalk in the 8-channel WDM system are shown in Fig. 4 , in which there are shown optical spectra in the 0-th channel when all channels are transmitted (Fig. 4a), and in the single-channel $10 \mathrm{GHz}$ transmission of pseudo-random data (Fig. 4b). The wider spectrum in the WDM case is due to the mutual interaction of neighbouring channels via SPM and XPM.
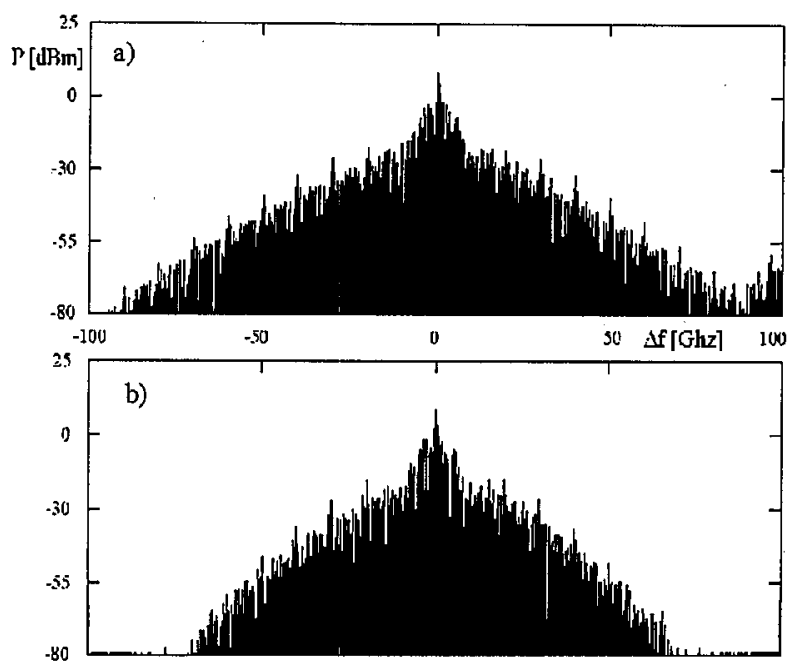

Fig. 4. Optical spectrum: (a) in the 0-th channel of the 8-channel WDM system, all channels are transmitted, (b) in single-channel $10 \mathrm{GHz}$ transmission of pseudo-random data as in the case (a).

In view of the above results, nonlinear interactions could cause severe limitations in optical transmission links. Possible ways to reduce nonlinear crosstalk are: a channel spacing increase, non-equal channel spacing, and a decrease in optical power:

\section{References}

[1] Y. Namihira, Electron. Lett. 30, 262 (1994).

[2] G.P. Agrawal, Nonlinear Fiber Optic, Academic Press, San Diego 1997.

[3] D. Marcuse, A.R. Chraplyvy, R.W. Tkacz, J. Lightwave Technol. 12, 885 (1994).

[4] G.P. Agrawal, Fiber-Optic Commnication Systems, Willey-Interscience Pub., New York 1992.

[5] W. Szczęsny, M. Marciniak, in: Intern. Conf. on Mathematical Methods in Electromagnetic Theory, Kharkov (Ukraine) 1998, p. 923.

[6] W. Szczęsny, M. Marciniak, in: Workshop COST P2 Applications of Non Linear Optical Phenomena, Limerick (Ireland) 1998, p. 16.

[7] P. Pietrzyk, W. Szczęsny, M. Marciniak, in: Worshop on Aplication of Nonlinear Optical Phenomena, Limerick (Ireland) 1998, p. 89.

[8] R.W. Tkach, A.R. Chraplyvy, F. Forghieri, A.H. Gnauck, R.M. Derosier, J. Lightwave Technol. 13, 841 (1995). 